\title{
Comparative Study on Educational Management in Public and Private Institutions in the Twin Cities of Pakistan
}

\author{
Muhammad Rizwan ${ }^{\mathrm{a} 1}$, Zeeshan Azad ${ }^{\mathrm{b}}$, Ashiq Ali $^{\mathrm{c}}$, Saba Mahmood ${ }^{\mathrm{d}}$ \\ ${ }^{a}$ School of Communication \& Information Engineering, Shanghai University, China, rizsoft@gmail.com, \\ 0000-0001-8903-5426 \\ ${ }^{\mathrm{b}}$ Shaheed Zulfiqar Ali Bhutto Institute of Science and Technology Islamabad, Pakistan, \\ chaudhary.zeeshan@gmail.com,0000-0002-1172-4894 \\ ${ }^{\mathrm{c}}$ School of Finance \& Management, Shanghai Normal University, China, ashiqali009@hotmail.com.0000- \\ 0003-1978-3247 \\ ${ }^{\mathrm{d}}$ School of Communication \& Information Engineering, Shanghai University, China, sabam777@gmail.com, \\ 0000-0003-2005-1932
}

\begin{abstract}
The aim of this research paper is to compare the quality of education delivered at the undergraduate level in the public and private institutions of the Twin Cities (Islamabad and Rawalpindi) in Pakistan. Data is collected with the help of questionnaires from 246 students. The data was collected from three public and three private educational institutions of the Twin Cities, and the age of students ranged from 18 to 27 years. The research instrument used in this paper is the independent sample t-test to find the difference between the three private and three public educational institutions selected in Rawalpindi and Islamabad in terms of different variables that are very important in measuring the quality of education. For analysis, Levene's test was adopted, which reflects the variations in educational quality with respect to academic staff availability in the twin cities. The differences in close supervision of students by academic staff between public and private sector educational institutions were also analysed, and the results reflected a difference between private and public educational institutions with respect to close supervision of students. The results also highlighted the factors that are involved in higher performance of students in private institutes as compared to that of students in public institutes. These factors can be implemented in the public sector to increase student performance.
\end{abstract}

Keywords: physical facilities, learning outcomes, student performance, undergraduate education

\section{Introduction}

This article focusses on the comparison of the quality of education provided in public and private education systems. Students have been treated as primary customers of higher education (Crawford, 1991), while employers, academic staff, government and families are considered to be secondary customers (Madu et al., 1994, Sirvanci, 1996). Previous studies in this area were conducted in India and were restricted to only three public institutes; therefore, we cannot generalise the findings for the entire educational policy. It can also be further extended to private and foreign universities to get better understanding of the differences between these different types of educational systems (Narang, 2012, p. 357) as this research is limited to only the public sector. Public schools are more common in rural India despite their inadequate performance and results (Muralidharan and Kremer, 2008).

There has been a great increase in the number of institutes in Pakistan; however, despite the sharp increase in private and public universities in Pakistan, the demand has been consistently higher than the supply. Students' enrolment has grown sharply because of the large number of youth, i.e. $63 \%$ of the population, is $<25$ years old. Another reason for increased demand is that people have understood the role of university education in achieving a better lifestyle similar to that in many developed and developing nations (Baum et al., 2010, Bloom et al., 2006). According to the Chairman of the Higher Education Commission (HEC), the enrolment in higher education of students in the age group of 18-

\footnotetext{
${ }^{1}$ Corresponding author: Muhammad Rizwan (rizsoft@gmail.com).
} 
23 years has increased up to three times, i.e. from 135,000 to 315,000 , which is more than double the percentage from 2.2 to $5.1 \%$ (Leghari, 2010). These statistics meet the target enrolment of all students in the age group of 18-23 years by the year 2010 . The number includes some 115,000 students who are studying in private universities and a quarter of these students are enrolled in higher education (Halai, 2011). This trend is followed in other countries in the Asian region as well as internationally. For example, close to half the students having higher education in Malaysia are enrolled in private institutions (Tierney and Sirat, 2008), and in India, private institutions have one-third of the overall enrolments in higher education (Agarwal, 2010). Almost half of the students having higher education in Latin American countries such as Brazil and Chile are enrolled in private universities (Altbach, 2008) and 23\% of the students in the USA are enrolled in private institutions (Tilak, 2006). According to estimations, by the year 2012, Pakistan accommodated about two million students at higher education levels and most of these students were accommodated in private institutions. Hence, the private universities are useful resources and have shown an ability to provide access to education for a larger number of students and are willing to expand this availability, reducing the burden on the public universities.

\section{Study objective}

This study compares the performances and results of public and private schools at a primary level. Salary packages offered at private schools are much less than those at public schools, have less pupil-teacher ratio and teachers love to be engaged in less multi-grade teaching; however, attendance of teachers is much better in private schools and more serious engagement in teaching activity is observed. Moreover, the private school teacher has higher ratio/probability of having a college degree and good qualification than a public school teacher. According to an Estonian research (Ploom and Haldma, 2012), measures of a school's operational performance management have great impacts on the satisfaction of other involved actors, such as teachers and parents. Therefore, schools' performance management system must ensure a balanced system wherein all levels - individual, operational and strategic performance - are in harmony or are synchronised well, leading to satisfaction for all the actors involved. The research focusses on the comparison of quality of education in public and private institutes on the basis of available physical facilities, academics, learning outcomes, responsiveness and personality development. This research will help the management and policymakers to determine the factors that are important regarding the quality of education and how we can improve this in the public sector. The purpose of this research is to improve the academic standards of Pakistan and compose one uniform education system for all the students. Right now we have thirty-one different boards and all of them have different syllabi, marking schemes and examiner reports.

\section{Significance of the study}

The policies used to operate educational organisations comprise educational management. There is no specific definition of educational management, as its development heavily depends on numerous disciplines, i.e. economics, political science, sociology etc. The definitions of educational management provided by different researchers and writers are partial because they reflect the particular sense of the authors. Educational management is actually the administration of obtainable educational establishments and systems. In Pakistan, there are two types of educational systems, i.e. public system and private system. According to Halai (2013), in Pakistan, private universities are those universities that are not operated by the Government of Pakistan but they receive support from the Government in the form of scholarships and student loans. Private universities are further divided into two groups: one is the for-profit institution and the other one is known as a non-profit institution. The majority of the private institutes are for-profit institutions. The study holds important implications as it will investigate these private institutes according to the variables mentioned and will help to design a framework applicable to the public institutes. This will allow the government to adopt a uniform educational system for everyone in the country.

\section{Research methodology}

According to Marshall and Rossman (1989, pp. 19), qualitative research can achieve the best "by emphasizing the promise of quality, depth and richness in the research finding". In order to collect data for this study, a survey method approach was adopted. The research questions were categorised into five sections, which were (a) physical facilities, (b) academics, (c) learning outcomes, (d) responsiveness and (e) personality development. The data was then arranged and organised to conduct certain statistical tests such as the independent sample $t$-test and Levene's test. Therefore, the study is a mix of both qualitative and quantitative analyses to ensure that the most relevant and accurate results are achieved.

Table 1: Review of key factors included in the questionnaire

\begin{tabular}{ll}
\hline Category & Factors included in the questionnaire \\
\hline Physical facilities & Technology, infrastructure, learning sources
\end{tabular}


Academics

Learning outcomes

Responsiveness

Personality development
Academic staff, training and development, course design

Learning techniques, course structure, extracurricular activities, social obligation

Services, systematic procedures, rules and regulations

Sports, cultural activities, recognition, knowledge enhancement

\section{Research contribution}

The research holds a significant contribution. The educational system of Pakistan is divided into two main segments, i.e. private and public sector schools. This comparative study analyses both sectors of education in the country and highlights the deficiencies present in the system. Educational policymakers can utilise this study to form a policy that can provide equally competent education to society. The study compares the factors such as physical facilities, academic facilities, learning outcomes, the responsiveness of the students and the personality development of students. This comparison will allow figuring out the deficiencies of the system and provide suggestions for improvement. Both systems have some advantages and disadvantages, and the comparison will allow combining the advantages together for a better educational system in Pakistan.

\section{Quality of education}

Previous studies conducted in this area highlighted the rural-urban differences in terms of teachers, students, school characteristics and achievements of the primary school students using a national representative sample (Tayyaba, 2012). This research overcomes the limitations of the previous research by the author by using an adequate sample in terms of proportion to address the potential disparities in achievements of rural and urban students and by determining how schooling, students and teacher-related factors reflect the differences in achievement and thus mainly addresses the issues of non-representative samples and insufficient sampling techniques. The classification of rural and urban schools was based on the geographical features of the school location listed by the national database of National Educational Management Information Systems (NEMIS), which considers the metropolitan statistical areas, population and distance from the road to assess the rural nature of a school. Data has been sampled using the probability proportional to size (PPS) systematic method and classroom sampled with an equal probability sampling. The multivariate analysis demonstrates that rural students performed better in Baluchistan and Sindh, while urban students outshined rural students in Punjab province. There was no statistically significant difference in the achievement of rural and urban students in the North-West Frontier Province. Hence, the results do not help the traditional conjecture that nationwide rural students are at a disadvantage in terms of quality of education, at least as reflected in their performance on four standardised tests.

Halai (2013) provided a developmental analysis of education in private sector universities in Pakistan as opposed to the public universities. In addition, the aim is to comprehend the quality of education in private universities keeping in mind the 2012 ranking data of the HEC, Pakistan. This method analyses and presents available statistics and publications on the HEC website on university education in the private and public sectors in three areas: development, availability and ranking in Pakistan and provides a comparison between them. The HEC, in 2002, introduced a system of ranking for all public and private universities operating within Pakistan. The first such exercise was conducted in 2006. The HEC Quality Assurance Committee, through a consultative process for the development of ranking methodology, analysed international ranking systems. The purpose of the ranking was to "share with the general public comprehensive data on key issues that determine the state of higher education in a particular institution". A handful of good-quality institutions in the private sector cover the substandard quality of education offered by a large number of private sector institutions. There are serious social consequences because of this condition of affairs as a considerable number of youth, in spite of investing fruitful years of their life and a big amount of money, do not acquire job skills in the specific area in which they are given training.

The universities in the private sector have done capacity building for enrolment of a larger number of students and have helped in making the enrolment double in the age bracket of 18-23 years. Despite the quality and variety of programmes offered at these institutions, there are areas in which private education needs to work really hard to come at par with the public sector. The author suggested that the quality of higher education in private universities can be made better by providing attractive incentives such as student scholarships and research funds to those private universities that have shown consistency in maintaining quality and an improving trend in the HEC rankings. The development of proper standards is a basic requirement for the assessment of quality in education. It is recognised that the term "standards" has not been used the same way in education-related discussions. Griffith (2008), on the basis of results clearly stated in educational standards, proposed a framework to assess the quality of education. Relative achievement assessment is concerned with how well the internal processes are working to move students towards accomplishing the outcomes established in the standards, and absolute achievement assessment, on the other hand, is concerned with the assessment of the extent to which those outcomes defined in the standards have been met by students. The study points 
to a number of assessments to illustrate the concept. These include those of the National Adaptation Plan (NAP) in Jamaica conducted at a national level, the Caribbean Examinations Council (CXC) conducted in countries within the Caribbean region and the Third International Mathematics and Science Study (TIMSS) conducted in several countries against established international standards.

\section{Data analysis and findings}

\subsection{Physical facilities}

In this comparative study, we have compared the results of physical facilities in the two types of education systems in Pakistan, the public sector and the private sector. In the context of physical facilities, there are eight different dimensions.

i. H0: There is no difference in terms of state-of-the-art technology between public sector and private sector educational institutions. The output table shows that the sample size is 124 and 122 for private and public sector educational institutions, respectively. The mean output is 2.88 and 2.67 for private and public institutions, respectively. The group statistics shows that the difference of means is 0.21 . To test whether the value 0.21 is statistically significant, we use Levene's test of variance, wherein $F=0.327$, with significance of 0.568 . H0 represents no difference between variances. Here, $t=1.367(<2)$, and the mean difference 0.21 is statistically not significant. This is confirmed by the significance $(0.173)$, which is $>0.05$. This means that with respect to the state-of-the-art technology in physical facilities, there is no difference between the six private and public educational institutions selected in Rawalpindi and Islamabad.

ii. H0: There is no difference in terms of the adequacy of facilities to render service between public sector and private sector educational institutions. The output table shows that sample size is 124 and 122 for private and public sector educational institutions, respectively. The mean output is 3.07 and 2.85 for private and public sector educational institutions, respectively. The group statistics shows that the difference of means is 0.22 . To check whether the value 0.22 is statistically significant, we use Levene's test of variance, where $F=0.806$, with significance of 0.370 . H0 represents no difference between the variances. Here, $t=1.78(<2)$, and the mean difference is 0.22 , which is statistically not significant. This is confirmed by the significance $0.075(>0.05)$. This means that with respect to the availability of adequate facilities to render services in terms of physical facilities, there is no difference between the six private and public educational institutions selected in Rawalpindi and Islamabad.

iii. H0: There is no difference in terms of well-equipped computer laboratories between public sector and private sector educational institutions. The output table shows that sample size is 124 and 122 for private and public sector educational institutions, respectively. The mean output is 3.25 and 3.25 , respectively. The group statistics shows that difference of means is 0.003 . To confirm whether the value 0.003 is statistically significant, we use Levene's test of variance, where $F=0.70$, with significance of 0.40 . H0 represents no difference between variances. Here, $t=0.028(<2)$, and the mean difference 0.003 is statistically not significant. This is confirmed by the significance 0.978 , which is $>0.05$. This means that with respect to well-equipped computer laboratories in physical facilities, there is no difference between the six private and public educational institutions selected in Rawalpindi and Islamabad.

iv. H0: There is no difference in providing comprehensive learning source between public sector and private sector educational institutions. The output table shows that sample size is 124 and 122 for private and public sector educational institutions, respectively. The mean output is 3.38 and 3.19, respectively. The group statistics shows that difference of means is 0.19 . To find whether the value 0.19 is statistically significant, we use Levene's test of variance, where $F=6.03$, with significance of 0.15 . H0 represents no difference between variances. Here, $t$ $=1.43(<2)$, and the mean difference 0.19 is statistically not significant. This is confirmed by the significance 0.153 , which is $>0.05$. This means that with respect to comprehensive learning source in physical facilities, there is no difference between the six private and public educational institutions selected in Rawalpindi and Islamabad.

v. H0: There is no difference in academic, residential and recreational facilities between public and private sector educational institutions. The output table shows that sample size is 124 and 122 for private and public sector educational institutions, respectively. The mean output is 3.43 and 3.30 for private and public sector educational institutions, respectively. The group statistics shows that difference of means is 0.13 . To find whether the value 0.13 is statistically significant, we use Levene's test of variance, where $F=6.23$, with significance of 0.13 . H0 represents no difference between variances. Here, $t=0.98(<2)$, and the mean difference 0.13 is statistically not significant. This is confirmed by the significance 0.327 , which is $>0.05$. This means that with respect to academic, residential and recreational facilities in physical facilities, there is no difference between the six private and public educational institutions selected in Rawalpindi and Islamabad.

vi. H0: There is no difference in the aesthetic view of facilities between public sector and private sector educational institutions. The output table shows that sample size is 124 and 122 for private and public sector educational 
institutions, respectively. The mean output is 3.27 and 3.20 , respectively. The group statistics shows that the difference of means is 0.069 . To find whether the value 0.069 is statistically significant, we use Levene's test of variance, where $F=0.172$, with significance of 0.679 . H0 represents no difference between variances. Here, $t$ $=0.602(<2)$, and the mean difference $=0.069$, which is statistically not significant. This is confirmed by the significance 0.548 , which is $>0.05$. This means that with respect to the aesthetic view of facilities in physical facilities, there is no difference between the six private and public educational institutions selected in Rawalpindi and Islamabad.

vii. H0: There is no difference in training between public sector and private sector educational institutions. The output table shows that sample size is 124 and 122 for private and public sector educational institutions, respectively. The mean output is 3.19 and 3.25 , respectively. The group statistics shows that the difference of means is 0.06 . To check whether the value 0.06 is statistically significant, we use Levene's test of variance, where $F=0.586$, with significance of 0.445 . H0 represents no difference between variances. Here, $t=-0.404$ $(<2)$, and mean difference is 0.06 , which is statistically not significant. This is confirmed by the significance 0.686 , which is $>0.05$. This means that with respect to training in well-equipped physical facilities, there is no difference between the six private and public educational institutions selected in Rawalpindi and Islamabad.

viii. H0: There is no difference in effective classroom management between public sector and private sector educational institutions. The output table shows that sample size is 124 and 122 for private and public sector educational institutions, respectively. The mean output is 3.16 and 3.48, respectively. The group statistics shows that difference of means $=0.314$. To find whether the value 0.314 is statistically insignificant, we use Levene's test of variance, where $F=5.18$, with significance of 0.024 . H0 represents that the difference between variances is assumed. Here, $t=2.029(>2)$, and the mean difference 0.314 is statistically significant. This is confirmed by the significance 0.04 , which is $<0.05$. This means that with respect to effective classroom management in physical facilities, there is a difference between the six private and public educational institutions selected in Rawalpindi and Islamabad.

\subsection{Academics}

The following section shows the comparative results of academics in the two types of education systems. In academics, there are six different dimensions.

i. H0: There is no difference in adherence to schedule between public and private sector educational institutions. The output table shows that sample size is 124 and 122 for private and public sector educational institutions, respectively. The mean output is 3.25 and 3.30 for private and public institutions, respectively. The group statistics shows that difference of means is 0.045 . To check whether the value 0.045 is statistically significant, we use Levene's test of variance, where $F=0.021$, with significance of 0.885 . H0 represents no difference between variances. Here, $t=0.335(<2)$, and mean difference $=0.045$, which is statistically not significant. This is confirmed by the significance 0.738 , which is $>0.05$. This means that with respect to adherence to schedule in academics, there is no difference between the six private and public educational institutions selected in Rawalpindi and Islamabad.

ii. H0: There is no difference in availability of enough subject teachers between public and private sector educational institutions. The output table shows that sample size is 124 and 122 for private and public sector educational institutions, respectively. The mean output is 3.66 and 3.17 for private and public institutions, respectively. The group statistics shows that difference of means is 0.49 . To find whether the value 0.49 is statistically significant, we use Levene's test of variance, where $F=17.74$, with significance of 0.000 . H0 represents that the difference between variances is assumed. Here, $t=3.547(>2)$, and mean difference $=0.49$, which is statistically significant. This is confirmed by the significance $=0.000(>0.05)$. This means that with respect to availability of enough subject teachers in academics, there is a difference between the six private and public educational institutions selected in Rawalpindi and Islamabad.

iii. H0: There is no difference in staff members available for consultation between public and private sector educational institutions. The output table shows that sample size is 124 and 122 for private and public sector educational institutions, respectively. The mean output is 3.65 and 3.35 for private and public institutions, respectively. The group statistics shows that the difference of means is 0.30 . To check whether 0.30 is statistically significant, we use Levene's test of variance where $F=3.12$ with significance of 0.078 . H0 represents the difference between variances is assumed. Here, $t=2.047(>2)$ and mean difference $=0.30$, which is statistically significant. This is confirmed by the significance $=0.042(<0.05)$. This means that with respect to the staff available for consultation in academics, there is a difference between the six private and public educational institutions selected in Rawalpindi and Islamabad.

iv. H0: There is no difference in close supervision of students' work between public and private sector educational institutions. The output table shows that sample size is 124 and 122 for private and public sector educational institutions, respectively. The mean output is 3.58 and 3.28 for private and public institutes, respectively. The group statistics shows that the difference of means is 0.30 . To check whether the value 0.30 is statistically 
significant, we use Levene's test of variance, where $F=8.396$ with significance of 0.004 . H0 represents that the difference between variances is assumed. Here, $t=2.246(>2)$, and mean difference $=0.30$, which is statistically significant. This is confirmed by the significance $=0.026(<0.05)$. This means that with respect to close supervision of students' work in academics, there is a difference between the six private and public educational institutions selected in Rawalpindi and Islamabad.

v. H0: There is no difference in expertise of teachers between public and private sector educational institutions. The output table shows that sample size is 124 and 122 for private and public sector educational institutions, respectively. The mean output is 3.97 and 3.58 for private and public institutions, respectively. The group statistics shows that the difference of means is 0.39 . To check whether 0.39 is statistically significant, we use Levene's test of variance where $F=41.124$ with significance of 0.000 . H0 represents that the difference between variances is assumed. Here, $t=2.694(>2)$, and mean difference $=0.39$, which is statistically significant. This is confirmed by the significance $=0.008 \quad(<0.05)$. This means that with respect to the expertise of teachers in academics, there is a difference between the six private and public educational institutions selected in Rawalpindi and Islamabad.

\subsection{Learning outcomes}

The following section shows the comparative results of learning outcomes between the two types of education systems. In the category learning outcomes, there are seven different dimensions.

i. H0: There is no difference in practical orientation between public and private sector educational institutions. The output table shows that sample size is 124 and 122 for private and public sector educational institutions, respectively. The mean output is 3.15 and 3.22 for private and public institutions, respectively. The group statistics shows that the difference of means is 0.07 . To find if 0.07 is statistically significant, we see Levene's test of variance, where $F=3.670$ with significance of 0.057 . H0 represents no difference between variances. Here, $t=-0.425(<2)$, and mean difference $=0.07$, which is statistically not significant. This is confirmed by the significance $=0.671(>0.05)$. This means that with respect to practical orientation in learning outcomes, there is no difference between the six private and public educational institutions selected in Rawalpindi and Islamabad.

ii. H0: There is no difference in demonstration of modern techniques between public and private sector educational institutions. The output table shows that sample size is 124 and 122 for private and public sector educational institutions, respectively. The mean output is 3.18 and 3.26 for private and public institutions, respectively. The group statistics shows that difference of means is 0.076 . To see if 0.076 is statistically significant, we use Levene's test of variance, where $F=11.834$, with significance of 0.001 . H0 represents no difference between variances. Here, $t=-0.562(<2)$, and mean difference $=0.076$, which is statistically not significant. This is confirmed by the significance $=0.575(>0.05)$. This means that with respect to demonstration of modern techniques in learning outcomes, there is no difference between the six private and public educational institutions selected in Rawalpindi and Islamabad.

iii. H0: There is no difference in designs of course structure between public and private sector educational institutions. The output table shows that sample size is 124 and 122 for private and public sector educational institutions, respectively. The mean output is 3.24 and 3.44 for private and public institutions, respectively. The group statistics shows that difference of means is 0.20 . To find whether 0.20 is statistically significant, we use Levene's test of variance, where $F=16.261$, with significance of 0.000 . H0 represents no difference between variances. Here, $t=-1.446(<2)$, and mean difference $=0.20$, which is statistically not significant. This is confirmed by the significance $=0.149(>0.05)$. This means that with respect to design of course structure in learning outcomes, there is no difference between the six private and public educational institutions selected in Rawalpindi and Islamabad.

iv. H0: There is no difference in problem-solving skills between public and private sector educational institutions. The output table shows that sample size is 124 and 122 for private and public sector educational institutions, respectively. The mean output is 3.25 and 3.23 for private and public institutions, respectively. The group statistics shows that difference of means is 0.01 . To see if 0.01 is statistically significant, we use Levene's test of variance where $F=3.575$ with significance of 0.060 . H0 represents no difference between variances. Here, $t$ $=0.089 \quad(<2)$, and mean difference $=0.01$, which is statistically not significant. This is confirmed by the significance $=0.929(>0.05)$. This means that with respect to problem-solving skills in learning outcomes, there is no difference between the six private and public educational institutions selected in Rawalpindi and Islamabad.

v. H0: There is no difference in extracurricular activities between public and private sector educational institutions. The output table shows that sample size is 124 and 122 for private and public sector educational institutions, respectively. The mean output is 3.04 and 3.08 for private and public institutions, respectively. The group statistics shows that difference of means is 0.04 . To see if 0.04 is statistically significant, we use Levene's test of variance where $F=4.194$ with significance of 0.042 . H0 represents no difference between variances. Here, $t=-0.244(<2)$, and mean difference $=0.04$, which is statistically not significant. This is confirmed by the 
significance $=0.808(>0.05)$. This means that with respect to extracurricular activities in learning outcomes, there is no difference between the six private and public educational institutions selected in Rawalpindi and Islamabad.

\subsection{Responsiveness}

The following section shows the comparative results of responsiveness between the two types of education systems. In responsiveness, there are four different dimensions.

i. H0: There is no difference in time response between public and private sector educational institutions. The output table shows that sample size is 124 and 122 for private and public sector educational institutions, respectively. The mean output is 2.88 and 2.95 for private and public institutions, respectively. The group statistics shows that difference of means is 0.07 . To check if 0.07 is statistically significant, we use Levene's test of variance, where $F=8.127$, with significance of 0.005 . H0 represents no difference between variances. Here, $t$ $=-0.470(<2)$, and mean difference $=0.07$, which is statistically not significant. This is confirmed by significance $=0.639(>0.05)$. This means that with respect to time response in responsiveness, there is no difference between the six private and public educational institutions selected in Rawalpindi and Islamabad.

ii. H0: There is no difference in staff help between public and private sector educational institutions. The output table shows that sample size is 124 and 122 for private and public sector educational institutions, respectively. The mean output is 3.14 and 3.44 for private and public institutions, respectively. The group statistics shows that difference of means is 0.29 . To check if 0.29 is statistically significant, we use Levene's test of variance where $F=8.796$, with significance of 0.003 . H0 represents that the difference between variances is assumed. Here, $t=2.014(>2)$, and mean difference $=0.29$, which is statistically significant. This is confirmed by the significance $=0.045(<0.05)$. This means that with respect to staff help in responsiveness, there is a difference between the six private and public educational institutions selected in Rawalpindi and Islamabad.

iii. H0: There is no difference in systematic procedure between public and private sector educational institutions. The output table shows that sample size is 124 and 122 for private and public sector educational institutions, respectively. The mean output is 3.27 and 3.11 for private and public institutions, respectively. The group statistics shows that difference of means is 0.16 . To see if 0.16 is statistically significant, we see Levene's test of variance, where $F=0.540$ with significance of 0.463 . H0 represents no difference between variances. Here, $t$ $=1.191(<2)$, and mean difference $=0.16$, which is statistically not significant. This is confirmed by the significance $=0.235(>0.05)$. This means that with respect to systematic procedure in responsiveness, there is no difference between the six private and public educational institutions selected in Rawalpindi and Islamabad.

iv. H0: There is no difference in official procedure being transparent between public and private sector educational institutions. The output table shows that sample size is 124 and 122 for private and public sector educational institutions, respectively. The mean output is 3.36 and 3.27 for private and public institutions, respectively. The group statistics shows that difference of means is 0.08 . To see if 0.08 is statistically significant, we use Levene's test of variance where $F=3.638$, with significance of 0.058 . H0 represents no difference between variances. Here, $t=0.622(<2)$, and mean difference $=0.08$, which is statistically not significant. This is confirmed by the significance $=0.535(>0.05)$. This means that with respect to official procedure being transparent in responsiveness, there is no difference between the six private and public educational institutions selected in Rawalpindi and Islamabad.

\subsection{Personality development}

The following section shows the comparative results of personality development between the two types of education systems. In personality development, there are three different dimensions.

i. H0: There is no difference in encouragement for sports and culture between public and private sector educational institutions. The output table shows that sample size is 124 and 122 for private and public sector educational institutions, respectively. The mean output is 3.01 and 3.13 for private and public institutions, respectively. The group statistics shows that difference of means is 0.11 . To find if 0.11 is statistically significant, we use Levene's test of variance where $F=1.894$ with significance of 0.170 . H0 represents no difference between variances. Here, $t=-0.678(<2)$, and mean difference $=0.11$, which is statistically not significant. This is confirmed by significance $=0.498(>0.05)$. This means that with respect to encouragement for sports and culture in personality development, there is no difference between the six private and public educational institutions selected in Rawalpindi and Islamabad.

ii. H0: There is no difference in enhancement of knowledge between public and private sector educational institutions. The output table shows that sample size is 124 and 122 for private and public sector educational institutions, respectively. The mean output is 3.58 and 3.36 for private and public institutions, respectively. The group statistics shows that difference of means is 0.21 . To find if 0.21 is statistically significant, we use Levene's test of variance, where $F=1.756$, with significance of 0.186 . H0 represents no difference between 
variances. Here, $t=1.755(<2)$ and mean difference $=0.21$, which is statistically not significant. This is confirmed by the significance $=0.081(>0.05)$. This means that with respect to enhancement of knowledge in personality development, there is no difference between the six private and public educational institutions selected in Rawalpindi and Islamabad.

iii. H0: There is no difference in recognition of the student between public and private sector educational institutions. The output table shows that sample size is 124 and 122 for private and public sector educational institutions, respectively. The mean output is 3.29 and 3.35 for private and public institutions, respectively. The group statistics shows that difference of means is 0.05 . To find if 0.05 is statistically significant, we use Levene's test of variance, where $F=1.559$, with significance of 0.213 . H0 represents no difference between variances. Here, $t=-0.400(<2)$ and mean difference $=0.05$, which is statistically not significant. This is confirmed by the significance $=0.689(>0.05)$. This means that with respect to recognition of the student in personality development, there is no difference between the six private and public educational institutions selected in Rawalpindi and Islamabad.

\section{Conclusion and recommendations}

Almost all the major concerns were covered in the study and the results showed that private institutes are better than public institutes in almost all aspects. To check whether there is any difference in effective classroom management between the public and private sectors, a sample size of 124 and 122 for private and public sector educational institutions, respectively, was taken. Mean output was checked for statistical insignificance with Levene's test of variance, and results showed that with respect to effective classroom management in physical facilities there is a difference between the six private and public educational institutions selected in Rawalpindi and Islamabad. The difference in availability of sufficient number of subject teachers between public and private sector educational institutions was also measured in the same samples and tested, and the results showed that with respect to the availability of enough subject teachers in academics, there is a difference between the six private and public educational institutions selected in Rawalpindi and Islamabad.

The difference in expertise of teachers between public and private sector educational institutions was also a subject of inquiry. The output table shows that with respect to expertise of teachers in academics, there is a difference between the six private and public educational institutions, as also with respect to staff help in responsiveness, selected in Rawalpindi and Islamabad. This study is basically about two different types of education systems in Pakistan. In this study, we contacted three public universities and three private universities. In order to get a clearer result, the population size can be increased by involving large number of institutes in both the public and private sectors. The same study can be conducted by considering religious institutes as one group and other education systems as the second group. The medium of study has variations in Pakistan, so a further research on this pattern can also be arranged.

There are many other fields that can be covered in such kind of study. There are many other concerns that can play a great role in the betterment of education in Pakistan. Some of the points that should be added in such a research are inquiries about the difference in the syllabi being taught at public and private institutes. There are many problems in the overall educational setup in Pakistan. There is a low level of literacy among the population and the standard of education is also low. The curriculum and related pedagogy are not appropriate or, at least, less than the set goals in many fields. Moreover, there is no planned system in which one step leads to the next and enables a student to develop a strong base for the field he/she is interested in. Moreover, even at higher educational levels, there is no such method to guide a student in bringing forward his or her potential or in deciding a suitable career. There are many different systems of educations working in Pakistan, resulting in social division and conflict. For instance, we have, in Pakistan, English medium schools, Urdu medium schools, and religious madrasas. Students studying in English medium schools and good private schools have very little awareness of religion and cultural roots, whereas students passing out from Urdu medium schools usually work at lower-level positions. Religious madrasas produce yet another class of people who are usually unaware of modern studies; they usually have strong sectarian biases and equal-to-none training in modern disciplines.

The government should declare education as the highest priority. We must know that unless illiteracy and lack of education are eradicated, the path to democracy will remain unknown, encompassing the danger of exploitation of the majority by the few, in the absence of political willingness among the ruling authorities to do something good in this area, because there is no matter more important to the betterment of a nation than human resource. It should be made mandatory for the government at all levels to take steps promoting various educational institutions according to national requirements. It should be made mandatory for various degree programmes that the candidates, after taking the final examinations, shall spend a period of time in teaching at assigned institutions to get a final degree.

\section{References}

Agarwal, P. (2007). Higher education in India: Growth, concerns and change agenda. Higher Education Quarterly, 61(2), 197-207. 
Altbach, P. G. (Ed.). (1999). Private Prometheus: private higher education and development in the 21 st century (No. 77). Greenwood Publishing Group.

Bevan, D., \& Kipka, C. (2012). Experiential learning and management education. Journal of Management Development, 31(3), 193-197.

Bloom, D. E., Hartley, M., \& Rosovsky, H. (2006). Beyond private gain: The public benefits of higher education. In International handbook of higher education (pp. 293-308). Springer Netherlands.

Brandon-Jones, A., Piercy, N., \& Slack, N. (2012). Bringing teaching to life: exploring innovative approaches to operations management education. International Journal of Operations \& Production Management, 32(12), 13691374.

Crawford, F. (1991), “Total quality management”, Committee of Vice-Chancellors and Principals, London, occasional paper, December.

Halai, N. (2013). The quality of private universities in Pakistan: An analysis of higher education commission rankings 2012. International Journal of Educational Management, 27(7), 775-786.

Halai, N. (2011). Private higher education in Pakistan. International Higher Education, 64, 21.

Leghari, J. (2010), "Interview Javaid R. Leghari: Chairperson HEC", available at: www.newslinemagazine.com/2010/10/interview-dr-javaid-r-laghari-chairperson-hec/(Accessed on August 10, 2016).

Madu, C. N., Kuei, C. H., \&Winokur, D. (1994). Total quality management in the university: a quality code of honor. Total Quality Management, 5(6), 375-390.

Muralidharan, K., \& Kremer, M. (2006).Public and private schools in rural India.Harvard University, Department of Economics, Cambridge, MA.

Narang, R. (2012). How do management students perceive the quality of education in public institutions?.Quality Assurance in Education, 20(4), 357-371.

Ploom, K and Haldma, T. (2012), "Balanced performance management in the public education system", Baltic Journal of Management, Vol. 8 No. 2, pp. 183-207

PROBE (1999), Government Report on Basic Education in India, Oxford University Press,New Delhi.

Sirvanci, M. (1996). Are students the true customers of higher education?.Quality Progress, 29(10), 99-104.

Tayyaba, S. (2012). Rural-urban gaps in academic achievement, schooling conditions, student, and teachers' characteristics in Pakistan. International Journal of Educational Management, 26(1), 6-26.

Tierney, W., \&Sirat, M. (2008). Challenges facing Malaysian higher education. International Higher Education, 53, 2324.

Tilak, J.B.G. (2006), Private Higher Education: Philanthropy to Profits, Higher Education in the World 200, University of Barcelona, UP Commons, Barcelona.

UNICEF. (2000). Defining Quality in Education. A paper presented by UNICEF at the meeting of The International Working Group on Education Florence, Italy, June 2000 (p. 3). Preface of the Working Paper. Document No. $\mathrm{UNICEF} / \mathrm{PD} / \mathrm{ED} / 00 / 02$. 